\title{
Endoscope-assisted middle fossa craniotomy for resection of inferior vestibular nerve schwannoma extending lateral to transverse crest
}

\author{
Adam N. Master, MD, ${ }^{1}$ Daniel S. Roberts, MD, ${ }^{1}$ Eric P. Wilkinson, MD, ${ }^{1}$ William H. Slattery, MD, ${ }^{1}$ and \\ Gregory P. Lekovic, MD, PhD²
}

Departments of ${ }^{1}$ Neurotology and ${ }^{2}$ Neurosurgery, House Clinic, Los Angeles, California

OBJECTIVE The authors describe their results using an endoscope as an adjunct to microsurgical resection of inferior vestibular schwannomas (VSs) with extension into the fundus of the internal auditory canal below the transverse crest.

METHODS All patients who had undergone middle fossa craniotomy for VSs performed by the senior author between September 2014 and August 2016 were prospectively enrolled in accordance with IRB policies, and the charts of patients undergoing surgery for inferior vestibular nerve tumors, as determined either on preoperative imaging or as intraoperative findings, were retrospectively reviewed. Age prior to surgery, side of surgery, tumor size, preoperative and postoperative pure-tone average, and speech discrimination scores were recorded. The presence of early and late facial paralysis, nerve of tumor origin, and extent of resection were also recorded.

RESULTS Six patients (all women; age range 40-65 years, mean age 57 years) met these criteria during the study period. Five of the 6 patients underwent gross-total resection; 1 patient underwent a near-total resection because of a small amount of tumor that adhered to the facial nerve. Gross-total resection was facilitated using the operative endoscope in 2 patients (33\%) who were found to have additional tumor visible only through the endoscope. All patients had a HouseBrackmann facial nerve grade of II or better in the immediate postoperative period. Serviceable hearing (American Academy of Otolaryngology-Head and Neck Surgery class A or B) was preserved in 3 of the 6 patients.

CONCLUSIONS Endoscope-assisted middle fossa craniotomy for resection of inferior vestibular nerve schwannomas with extension beyond the transverse crest is safe, and hearing preservation is feasible.

https://thejns.org/doi/abs/10.3171/2017.12.FOCUS17663

KEY WORDS vestibular schwannoma; endoscope; middle fossa craniotomy

$\mathrm{T}$ UMORS arising from the cerebellopontine angle (CPA) account for $10 \%$ of intracranial tumors. By far the most common pathology of the CPA is vestibular schwannoma (VS), comprising $70 \%-90 \%$ of CPA masses. Patients most often present with poor hearing and tinnitus on the affected side, but some have serviceable hearing (i.e., American Academy of OtolaryngologyHead and Neck Surgery [AAO-HNS] hearing classification A or B). Patients presenting with serviceable hearing at our institution are given the opportunity to undergo hearing-conservation surgery.

The 2 available approaches for resection of VS that have the potential for hearing preservation are retrosigmoid (RS) and middle fossa craniotomy (MFC). The RS approach is versatile and allows the removal of small to large VSs from the CPA and internal auditory canal (IAC). Its disadvantages for hearing conservation surgery include limited exposure of the lateral IAC without disrupting the inner ear and sacrificing hearing, leading to a lower hearing preservation rate for RS surgery compared with MFC.

The MFC technique was first described by William House in 1961 for the removal of VSs. ${ }^{5}$ Unlike the RS approach, it offers access to almost the entire IAC, from porus to fundus, without entering the inner ear. MFC is best

ABBREVIATIONS AAO-HNS = American Academy of Otolaryngology-Head and Neck Surgery; $A B R=$ auditory brainstem response; $C P A=$ cerebellopontine angle; $I A C=$ internal auditory canal; $M F C=$ middle fossa craniotomy; PTA = pure-tone average; $R S=$ retrosigmoid; $S D S=$ speech discrimination score; VS = vestibular schwannoma. SUBMITTED October 31, 2017. ACCEPTED December 11, 2017. INCLUDE WHEN CITING DOI: 10.3171/2017.12.FOCUS17663. 
used in cases of intracanalicular VSs smaller than $2.5 \mathrm{~cm}$. This approach yields higher rates of hearing preservation in experienced hands than an RS approach does. . $^{3,9,13,15,16}$

Although MFC provides access to the fundus of the IAC, visualization inferior to the transverse crest is limited by the poor angle of dissection the approach requires. ${ }^{2}$ Tumors that extend to the far-lateral part of the IAC can also extend inferior to the transverse crest, and their full extension might not be appreciated through a conventional microscopic approach. Traditionally, the surgeon has had to blindly dissect this part of the tumor, which can result in incomplete removal and damage to the cochlear nerve. Unsurprisingly, hearing preservation is poorer in these instances. $^{3}$

As a way of overcoming this limitation, we recently reported using an endoscope as a way to view the area inferior to the transverse crest and as an aid for dissection under endoscopic guidance. ${ }^{1}$ The endoscope provides a wider viewing angle than the microscope, and, with the viewpoint arising from the tip of the scope, the surgeon can view around corners, especially with an angled endoscope. In the current study, we describe our results using the endoscope as an adjunct to microsurgical resection of inferior VSs with extension into the fundus of the IAC below the transverse crest. To accomplish this, we employed the endoscope prospectively with all patients undergoing MFC for VS during the study period at our institution.

\section{Methods}

\section{Patient Population}

Patients were included in this study if they had a primary diagnosis of VS arising from the inferior vestibular nerve (as determined either by preoperative imaging or intraoperative findings) and met criteria for MFC at our institution between September 2014 and August 2016. Patients are eligible for MFC at our institution if the tumor is less than $2.5 \mathrm{~cm}$ (measured in the transverse plane, including the IAC component) and is located in the IAC or has limited CPA extension not contacting the brainstem, and the patient is 65 years or younger and has serviceable hearing (AAO-HNS class A or B). Six patients (all female) ranging in age from 40 to 65 years (mean age 57 years) met these criteria during the study period. All had sporadic VS (i.e., patients with neurofibromatosis type 2 were excluded). Patient age prior to surgery, side of surgery, tumor size, preoperative pure-tone average (PTA), and speech discrimination scores (SDSs) were recorded. Postoperative PTA, SDS, presence of early and late facial paralysis, nerve of tumor origin, and extent of resection were all recorded postoperatively. The study was conducted in accordance with IRB policies (St. Vincent Medical Center IRB).

\section{Surgery}

All patients underwent surgery performed by a single neurosurgeon (G.P.L.) at a single institution. Each patient underwent a standard MFC for exposure of the IAC. After identification of the facial nerve at the fundus and labyrinthine segment, the surgeon opened the posterior fossa dura, and CSF was drained. The tumor was further dissected without the use of a fixed retractor and following standardized microsurgical techniques. Patients were monitored during surgery with intraoperative auditory brainstem responses (ABRs).

\section{Endoscope Assistance}

Following microsurgical removal of the tumor and with the aid of the endoscope, the operating surgeon assessed whether any additional tumor was likely to be found. The surgical endoscope was introduced into the field under microscopic guidance to ensure safe entry. A standard 3-mm, 14-cm rigid $70^{\circ}$ endoscope (Storz) was used with each patient to obtain a view of the most lateral aspect of the fundus. Specifically, the area inferior to the transverse crest was viewed, which often cannot be seen entirely with a microscope. Using the endoscope for access, residual tumor presence not seen with the microscope was determined. If residual tumor was present, endoscopic removal was attempted using standard microsurgical instruments. Any change in management was recorded, along with whether the endoscope allowed further dissection. In addition, it was recorded whether any complications had been prevented by virtue of the increased view provided by the endoscope.

\section{Results}

\section{Illustrative Case}

Case 6

This 65-year-old woman was initially diagnosed with an 8-mm left-sided inferior vestibular nerve schwannoma after presenting with dizziness (Fig. 1A and B). Videonystagmography revealed a $16 \%$ left-sided weakness. The patient's hearing was not affected by the tumor (SDS 100\%, PTA $6 \mathrm{~dB}$ ). Her dizziness resolved, and she elected to monitor the tumor with serial MRI. Over the following 3 years, the patient's tumor increased in size to $15 \mathrm{~mm}$ with impaction of tumor at the fundus (Fig. 1C and D), and the patient had mild progression of hearing loss (SDS 84\%, PTA $16 \mathrm{~dB}, \mathrm{AAO}-\mathrm{HNS}$ class A), at which time she elected to undergo hearing preservation surgery. She underwent an endoscope-assisted left-sided MFC with successful resection of tumor with no significant change in her SDS but mild increase in her PTA (SDS 88\%, PTA $32 \mathrm{~dB}$, AAOHNS class B). Postoperative MRI performed 1 year after surgery confirmed gross-total resection without evidence of tumor recurrence (Fig. 1E).

\section{Patients}

Data from all 6 patients included in this study are summarized in Table 1. All 6 patients successfully underwent MFC for removal of a VS arising from the inferior vestibular nerve. Tumor size ranged from 7 to $20 \mathrm{~mm}$ (mean size $14 \mathrm{~mm}$ ). Patients underwent both clinical (range 1-30 months, median 9 months) and MRI (range 3-28 months, median 12 months) follow-up.

Five of the 6 patients underwent gross-total resection; 1 patient underwent a near-total resection due to a small amount of tumor adherent to the facial nerve. Gross-total resection was facilitated using an operative endoscope in 

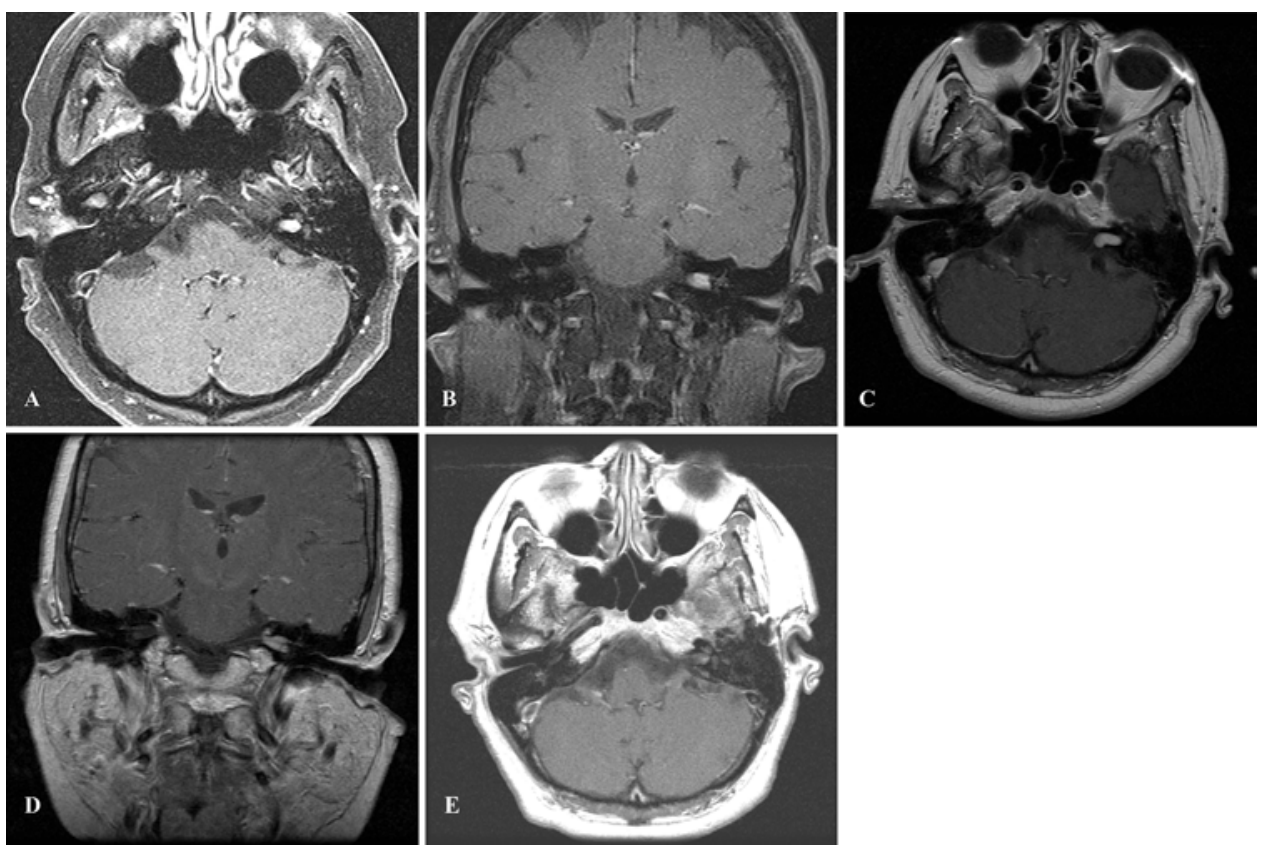

FIG. 1. Axial (A) and coronal (B) T1-weighted, Gd contrast-enhanced MR images demonstrating an enhancing tumor of the left CPA extending into the fundus of the IAC inferior to the transverse crest. Follow-up axial (C) and coronal (D) images demonstrating growth of the tumor prior to surgery. Postoperative axial $\mathrm{Gd}$ contrast-enhanced image $(\mathrm{E})$ demonstrating the absence of any residual tumor.

2 patients (33\%) who were found to have additional tumor visible only through the endoscope. In one patient this was anticipated, and in the other the finding of residual tumor was unexpected. The remaining tumor was removed using endoscope assistance.

All patients had a House-Brackmann facial nerve grade of II or better in the immediate postoperative period, and all patients had House-Brackmann grade I at the time of last follow-up (mean 12 months, range 1-30 months).

Preoperatively, hearing in all patients was graded as AAO-HNS class A. Hearing was preserved in 3 patients postoperatively (class A or B); 1 patient had nonserviceable hearing (40\% SDS and $39 \mathrm{~dB}$ PTA, class D) after surgery, and 2 patients had no measureable audiometric response to sound after surgery.

Auditory brainstem response (ABR) monitoring was used in all surgeries to monitor the cochlear nerve. The ABR signal was lost in 2 patients and delayed in 1; the re- maining 3 patients had stable ABRs. Intraoperative ABR changes were correlated with postoperative hearing loss.

\section{Complications}

One patient had delayed facial paralysis beginning on postoperative day 5, which progressed to House-Brackmann grade III. At the final postoperative visit, it had recovered to grade I. Other complications included cranial neuropathy other than hearing loss (abducens nerve palsy and V3 numbness in 1 case each), and CSF leak requiring lumbar drainage (1 case). The abducens nerve palsy resolved by the 8-month follow-up, but the V3 numbness persisted to light touch and temperature as of last followup (30 months). There were no deaths.

\section{Discussion}

MFC for removal of VS is a safe and effective proce-

TABLE 1. Clinical and hearing characteristics of patients

\begin{tabular}{|c|c|c|c|c|c|c|c|c|c|c|c|}
\hline \multirow{2}{*}{$\begin{array}{l}\text { Case } \\
\text { No. }\end{array}$} & \multirow{2}{*}{$\begin{array}{l}\text { Age } \\
\text { (yrs) }\end{array}$} & \multirow{2}{*}{$\begin{array}{l}\text { MRI Follow-Up } \\
\text { (mos) }\end{array}$} & \multicolumn{2}{|c|}{ Tumor } & \multirow[b]{2}{*}{ EOR } & \multirow{2}{*}{$\begin{array}{l}\text { Preop } \\
\text { SDS }\end{array}$} & \multirow{2}{*}{$\begin{array}{c}\text { Preop } \\
\text { PTA (dB) }\end{array}$} & \multirow{2}{*}{$\begin{array}{l}\text { Postop } \\
\text { SDS }\end{array}$} & \multirow{2}{*}{$\begin{array}{l}\text { Postop } \\
\text { PTA (dB) }\end{array}$} & \multicolumn{2}{|c|}{ HB Facial Nerve Grade } \\
\hline & & & Side & Size $(\mathrm{mm})$ & & & & & & Early & Late \\
\hline 1 & 53 & 3 & Rt & 16 & GTR & $100 \%$ & 15 & NR & NR & II & I \\
\hline 2 & 57 & 28 & $\mathrm{Lt}$ & 20 & NTR & $84 \%$ & 29 & $40 \%$ & 39 & II & I \\
\hline 3 & 40 & 18 & $\mathrm{Lt}$ & 7 & GTR & $100 \%$ & 8 & $100 \%$ & 5 & II & 1 \\
\hline 4 & 66 & 12 & Lt & 17 & GTR & $80 \%$ & 20 & NR & NR & I & I \\
\hline 5 & 59 & 4 & $\mathrm{Lt}$ & 7 & GTR & $80 \%$ & 29 & $88 \%$ & 34 & I & I \\
\hline 6 & 65 & 12 & $\mathrm{Lt}$ & 15 & GTR & $84 \%$ & 16 & $88 \%$ & 32 & 1 & I \\
\hline
\end{tabular}

EOR = extent of resection; GTR = gross-total resection; $\mathrm{HB}=$ House-Brackmann; NR = not recorded; NTR = near-total resection. 
dure for removing small intracanalicular tumors while offering the potential for hearing preservation. However, tumors that are impacted in the far-lateral portion of the IAC below the transverse crest may not be entirely observable through the conventional microscopic approach and are considered to have poor prognosis for hearing preservation.

In this series, serviceable hearing was maintained in 3 of 6 patients $(50 \%)$ in a highly selected patient population with poor prognostic features for hearing preservation, namely inferior vestibular nerve tumor origin with tumor extending lateral to the transverse crest. This rate $(50 \%)$ is lower than that reported in larger trials by our institution and others for middle fossa approaches generally $(20 \%-$ 71\%). ${ }^{9,12,13,15}$ However, we believe that this rate is acceptable, given the poor prognosis for hearing preservation in this highly selected patient population.

The addition of the endoscope in the MFC approach helps the surgeon overcome some of these limitations. First, the view from the microscope is limited by surgical access. The position and angle of the surgeon's view are controlled by the steep angle of the middle fossa floor and the limitations of retraction on the temporal lobe. With a microscope, the view is restricted to a straight line of sight dictated by where the light of the microscope can be positioned. By contrast, the endoscope provides a wide-angle view from its tip and is not limited by other structures that block the surgeon's line of sight. Therefore, increased temporal lobe retraction and further bone removal are not required for a more lateral view. Second, the viewing angle of the microscope must remain at $0^{\circ}$, but endoscope tips can be moved by up to $70^{\circ}$, allowing extreme viewing angles to be achieved without changes to the surgeon's hand position.

There are few studies in the English-language literature that have examined endoscope-assisted VS surgery. The vast majority of studies examine the RS approach. Göksu et al. reported on 60 patients who had VSs removed through an endoscope-assisted RS approach. ${ }^{4}$ They found no change in the rate of hearing preservation, which was $24 \%$ in their series, but the patients did not have any residual or recurrent disease. Overall, they concluded that endoscopes help ensure total tumor resection.

Kumon et al. compared 28 patients who underwent endoscope-assisted RS VS removal with 43 patients who underwent conventional RS resection. ${ }^{8}$ There was no significant difference in preservation of useful hearing, facial nerve function, or tumor recurrence between the groups, but there was a significant difference in residual tumor in patients whose tumors were located beyond the midportion of the IAC. There was also a lower overall incidence of residual tumor in the endoscope group, although the difference was not significant.

Very few studies have examined the utility of endoscopes in MFC. Wackym et al. explored the IAC in 3 patients after tumor resection via an MFC and noted improved visualization of the fundus, but they did not use the endoscope for dissection. ${ }^{14}$ Our group previously reported our experience with 2 patients undergoing endoscopeassisted MFC. ${ }^{1}$ Recognizing the visual advantage of using the endoscope for dissection in the lateral fundus, we wanted to further examine whether endoscopy would be of use in treating this specific subset of tumors.

A larger case review is needed to draw significant conclusions about whether the use of endoscopy in MFC for VS could increase hearing preservation rates. Hypothetically, the improved visualization offered by the endoscope could aid surgeons in distinguishing cochlear nerve fibers from tumor. In this study, however, the endoscope was used after microsurgical resection damage to cochlear nerve fibers might already have occurred, on the introduction of the endoscope.

In general, facial nerve outcomes for resection of small VSs are favorable for any approach. ${ }^{11}$ No patient in this study had long-term facial nerve dysfunction. Other groups have reported an approximately 1-year rate of House-Brackmann grade I or II in $70 \%-100 \%$ of patients. ${ }^{6,7,10}$ The endoscope might provide an added degree of facial nerve protection when used for dissection because it allows further lateral visualization of the IAC. If the tumor involves this portion of the IAC, dissection from a conventional microscopic approach is often blind, which could put the facial nerve at risk.

Based on our experience, the endoscope may have a role in MFC for tumors that extend to the lateral portion of the fundus. Importantly, the surgeon's ability to estimate the extent of tumor resection is limited by poor visibility for tumors impacted laterally below the crest. Not only does the endoscope allow confirmation of the completeness of microscopic tumor resection, it allows further dissection of tumor not visible with the microscope. The endoscope does increase overall surgical time, however, and adds cost to the surgery. Furthermore, it decreases depth perception, and damage to critical structures can occur from contact with the endoscope. In this series, however, there were no complications from the use of the endoscope.

This study is limited by the biases normally seen in a case series. The limited number of patients may obscure the conclusions we drew from our patient data. In addition, there was no control group of patients with which to compare our results. Future studies should compare rates of recurrence or residual tumor among patients undergoing traditional microscope-only surgery.

\section{Conclusions}

Endoscope-assisted MFC for resection of inferior VSs with extension beyond the transverse crest is safe, and hearing preservation is feasible. The use of the endoscope can provide the surgeon with additional information about the completeness of the tumor resection and minimize blind dissection in the lateral IAC that may jeopardize hearing preservation. Further studies are needed to draw conclusions about whether endoscope use in MFC will lead to less recurrent or residual VS.

\section{References}

1. Chen BS, Roberts DS, Lekovic GP: Endoscopic-assisted middle fossa craniotomy for resection of vestibular schwannoma. J Neurol Surg Rep 77:e001-e007, 2016

2. Driscoll CL, Jackler RK, Pitts LH, Banthia V: Is the entire fundus of the internal auditory canal visible during the 
middle fossa approach for acoustic neuroma? Am J Otol 21:382-388, 2000

3. Goddard JC, Schwartz MS, Friedman RA: Fundal fluid as a predictor of hearing preservation in the middle cranial fossa approach for vestibular schwannoma. Otol Neurotol 31:1128-1134, 2010

4. Göksu N, Yilmaz M, Bayramoglu I, Aydil U, Bayazit YA: Evaluation of the results of endoscope-assisted acoustic neuroma surgery through posterior fossa approach. ORL J Otorhinolaryngol Relat Spec 67:87-91, 2005

5. House WF: Surgical exposure of the internal auditory canal and its contents through the middle, cranial fossa. Laryngoscope 71:1363-1385, 1961

6. Irving RM, Jackler RK, Pitts LH: Hearing preservation in patients undergoing vestibular schwannoma surgery: comparison of middle fossa and retrosigmoid approaches. J Neurosurg 88:840-845, 1998

7. Isaacson B, Telian SA, El-Kashlan HK: Facial nerve outcomes in middle cranial fossa vs translabyrinthine approaches. Otolaryngol Head Neck Surg 133:906-910, 2005

8. Kumon Y, Kohno S, Ohue S, Watanabe H, Inoue A, Iwata $\mathrm{S}$, et al: Usefulness of endoscope-assisted microsurgery for removal of vestibular schwannomas. J Neurol Surg B Skull Base 73:42-47, 2012

9. Kutz JW Jr, Scoresby T, Isaacson B, Mickey BE, Madden CJ, Barnett SL, et al: Hearing preservation using the middle fossa approach for the treatment of vestibular schwannoma. Neurosurgery 70:334-341, 2012

10. Meyer TA, Canty PA, Wilkinson EP, Hansen MR, Rubinstein JT, Gantz BJ: Small acoustic neuromas: surgical outcomes versus observation or radiation. Otol Neurotol 27:380-392, 2006

11. Noudel R, Gomis P, Duntze J, Marnet D, Bazin A, Roche $\mathrm{PH}$ : Hearing preservation and facial nerve function after microsurgery for intracanalicular vestibular schwannomas: comparison of middle fossa and retrosigmoid approaches. Acta Neurochir (Wien) 151:935-945, 2009

12. Slattery WH, Hoa M, Bonne N, Friedman RA, Schwartz MS, Fisher LM, et al: Middle fossa decompression for hearing preservation: a review of institutional results and indications. Otol Neurotol 32:1017-1024, 2011
13. Sughrue ME, Yang I, Aranda D, Kane AJ, Parsa AT: Hearing preservation rates after microsurgical resection of vestibular schwannoma. J Clin Neurosci 17:1126-1129, 2010

14. Wackym PA, King WA, Poe DS, Meyer GA, Ojemann RG, Barker FG, et al: Adjunctive use of endoscopy during acoustic neuroma surgery. Laryngoscope 109:1193-1201, 1999

15. Wang AC, Chinn SB, Than KD, Arts HA, Telian SA, ElKashlan HK, et al: Durability of hearing preservation after microsurgical treatment of vestibular schwannoma using the middle cranial fossa approach. J Neurosurg 119:131-138, 2013

16. Wilkinson EP, Roberts DS, Cassis A, Schwartz MS: Hearing outcomes after middle fossa or retrosigmoid craniotomy for vestibular schwannoma tumors. J Neurol Surg B Skull Base 77:333-340, 2016

\section{Disclosures}

The authors report no conflict of interest concerning the materials or methods used in this study or the findings specified in this paper.

\section{Author Contributions}

Conception and design: Lekovic. Acquisition of data: all authors. Analysis and interpretation of data: all authors. Drafting the article: all authors. Critically revising the article: all authors. Reviewed submitted version of manuscript: all authors. Approved the final version of the manuscript on behalf of all authors: Lekovic.

\section{Supplemental Information}

Current Affiliations

Dr. Roberts: Division of Otolaryngology, UConn Health, Farmington, CT.

\section{Correspondence}

Gregory P. Lekovic: House Clinic, Los Angeles, CA. glekovic@ houseclinic.com. 